\title{
Power System Reactive Power Optimization Based on Fuzzy Formulation and Interior Point Filter Algorithm
}

\author{
Zheng Fan ${ }^{1}$, Wei Wang ${ }^{2}$, Tian-jiao Pu ${ }^{1}$, Guang-yi Liu ${ }^{1}$, Zhi Cai ${ }^{1}$, Ning Yang ${ }^{3}$ \\ ${ }^{1}$ China Electric Power Research Institute, Beijing, China \\ ${ }^{2}$ Gansu Electric Power Corporation, Lanzhou, China \\ ${ }^{3}$ Northeast China Grid Company, Shenyang, China \\ Email: fzsxqs@163.com
}

Received March, 2013

\begin{abstract}
Considering the soft constraint characteristics of voltage constraints, the Interior-Point Filter Algorithm is applied to solve the formulation of fuzzy model for the power system reactive power optimization with a large number of equality and inequality constraints. Based on the primal-dual interior-point algorithm, the algorithm maintains an updating "filter" at each iteration in order to decide whether to admit correction of iteration point which can avoid effectively oscillation due to the conflict between the decrease of objective function and the satisfaction of constraints and ensure the global convergence. Moreover, the "filter" improves computational efficiency because it filters the unnecessary iteration points. The calculation results of a practical power system indicate that the algorithm can effectively deal with the large number of inequality constraints of the fuzzy model of reactive power optimization and satisfy the requirement of online calculation which realizes to decrease the network loss and maintain specified margins of voltage.
\end{abstract}

Keywords: Power System; Reactive Power Optimization; Fuzzy; Filter; Interior-point Algorithm; Online Calculation

\section{Introduction}

On the premise of safe and stable operation, the reactive power optimization of power system realizes hierarchical and regional balance of reactive power, improves voltage quality and reduces network loss by means of adjustment of the reactive power controllers such as terminal voltages of generators, tap positions of on load tap changers (OLTCs) and switchable shunt capacitor/reactors. The model of traditiona1 reactive power optimization is usually expressed as minimization of the active network loss under rigid voltage constraints which almost not considers the security margin of voltage and the characteristics of soft constraints when dealing with some voltage constraints. It makes the optimized voltage of some buses too close to their high limits, which becomes the threat of the system because the ability of enduring the variation is decreased remarkably $[1,2]$. Actually, the expected value of operating voltage is a fuzzy concept. The voltage constraints of load nodes for reactive power optimization are soft constraints. In [1-4], a mathematical model that is closer to the reality is established by introducing the fuzzy theory for dealing with soft constraints; the formulation of fuzzy model for reactive power optimization can decrease the network loss and maintain specified margins of voltage.

Now, the primal-dual interior-point algorithm is wide- ly used in the field of reactive power optimization [5-8], because it has the advantages of rapid convergence, strong robustness and insensitivity to the initial value [5]. The interior-point filter algorithm [9, 10] (IPFA) is the latest achievement in nonlinear optimization research. Based on the primal-dual interior-point algorithm, it maintains an updating "filter" at each iteration in order to decide whether to admit correction of iteration point which can avoid effectively oscillation due to the conflict between the decrease of objective function and the satisfaction of constraints and ensure the global convergence, Moreover, the "filter" improves computational efficiency because it filters the unnecessary iteration points [11, 12].

In this paper, the IPFA is applied to solve the formulation of fuzzy model for the power system reactive power optimization with a large number of equality and inequality constraints. The example of an actual power system indicates that the algorithm can effectively deal with the large number of inequality constraints of the fuzzy model of reactive power optimization and satisfy the requirement of online calculation which realizes to decrease the transmission loss and maintain specified margins of voltage.

The paper is organized as follows. In section 2 the formulation of fuzzy model for the power system reac- 
tive power optimization is introduced. In section 3 the solution of the model based on an interior point filter algorithm is presented. Test results of a practical system are reported in section 4 and conclusions are made in section 5 .

\section{Formulation of Fuzzy Model for Reactive Power Optimization}

The traditional mathematical model of reactive power optimization is established under a certain given active power dispatching mode by using the voltage amplitudes, phase angles, outputs of reactive power compensation device and transformation ratios of OLTC as decision variables and representing other variables in the form of function of decision variables. The problem can be described as follows:

$$
\begin{aligned}
& \min f(\mathbf{x}) \\
& \text { s.t. } \mathbf{g}_{1}(\mathbf{x})=\mathbf{0} \\
& \mathbf{g}_{2}(\mathbf{x})=\mathbf{0} \\
& \mathbf{h}_{\min } \leq \mathbf{h}(\mathbf{x}) \leq \mathbf{h}_{\max } \\
& \mathbf{h}_{\text {min }}^{\prime} \leq \mathbf{h}^{\prime}(\mathbf{x}) \leq \mathbf{h}_{\text {max }}^{\prime}
\end{aligned}
$$

where $\mathbf{x}=\left[\mathbf{x}_{1}^{\mathrm{T}}, \mathbf{x}_{2}{ }^{\mathrm{T}}, \mathbf{x}_{3}{ }^{\mathrm{T}}\right]^{\mathrm{T}} ; \mathbf{x}_{1}$ is the vector composed of terminal voltages of generators, outputs of reactive power compensation devices and transformation ratios of OLTCs; $\mathbf{x}_{2}$ is the vector composed of the voltages of PQ buses, it has the characteristics of soft constraint; $\mathbf{x}_{3}$ is the vector composed of voltage phase angles of all buses except slack bus; $f(\mathbf{x})$ is active network loss of power system; $\mathbf{g}_{1}(\mathbf{x})=\mathbf{0}$ are active balance equations( $\mathrm{N}$-1-dimensional, $\mathrm{N}$ is the number of nodes in the system); $\mathbf{g}_{2}(\mathbf{x})=\mathbf{0}$ are reactive balance equations( $\mathrm{N}-\mathrm{N}_{\mathrm{G}}$-dimensional, $\mathrm{N}_{\mathrm{G}}$ is the number of generators in the system); $\mathbf{h}(\mathbf{x})$ are the inequality constraints of terminal voltages and reactive power outputs of generators, outputs of reactive power compensation devices and transformation ratios of OLTCs, which are regarded as hard constraints; $\mathbf{h}^{\prime}(\mathbf{x})$ are the inequality constraints of voltages of PQ buses, which are regarded as soft constraints; $\mathbf{h}_{\min }$ and $\mathbf{h}_{\max }$ are the vectors of lower limits and upper limits of hard constraints respectively $\left(\mathrm{N}_{\mathrm{H}}\right.$-dimensional, $\mathrm{N}_{\mathrm{H}}$ is the sum of $2 \mathrm{~N}_{\mathrm{G}}, \mathrm{N}_{\mathrm{C}}$ and $\mathrm{N}_{\mathrm{T}}, \mathrm{N}_{\mathrm{C}}$ and $\mathrm{N}_{\mathrm{T}}$ are the number of reactive power compensation devices and OLTCs respectively); $\mathbf{h}_{\text {min }}^{\prime}$ and $\mathbf{h}_{\text {max }}^{\prime}$ are the vectors of lower limits and upper limits of soft constraints respectively ( $\mathrm{N}_{\mathrm{S}}$ dimensional, $\mathrm{N}_{\mathrm{S}}$ is the number of PQ buses).

Fuzzy theory is introduced for the justfication of the problem, and it is the key to select membership functions. In this paper, the membership functions for objective function and soft constraint variables are expressed as piecewise linear functions [1]. The membership function of objective function is represented as follows:

$$
\mu(f(\mathbf{x}))=\left\{\begin{array}{ll}
1 & f(\mathbf{x}) \leq f_{m}-\varepsilon_{p} \\
\frac{f_{m}-f(\mathbf{x})}{\varepsilon_{p}} & f_{m}-\varepsilon_{p}<f(\mathbf{x}) \leq f_{m} \\
0 & f(\mathbf{x})>f_{m}
\end{array}\right\}
$$

where $\varepsilon_{p}$ is the ideal maximum reduction of network loss; $f_{m}$ is maximum value of network loss.

The membership function of soft constraint variables is defined as follows:

$$
\left.\mu h_{i}^{\prime}(\mathbf{x})\right)=\left\{\begin{array}{cl}
1 & h_{i \min }^{\prime}+\varepsilon_{v i} \leq h_{i}^{\prime}(\mathbf{x}) \leq h_{i \max }^{\prime}-\varepsilon_{v i} \\
\frac{h_{i \max }^{\prime}-h_{i}^{\prime}(\mathbf{x})}{\varepsilon_{v i}} & h_{i \max }^{\prime}-\varepsilon_{v i}<h_{i}^{\prime}(\mathbf{x}) \leq h_{i \max }^{\prime} \\
\frac{h_{i}^{\prime}(\mathbf{x})-h_{i \min }^{\prime}}{\varepsilon_{v i}} & h_{i \min }^{\prime} \leq h_{i}^{\prime}(\mathbf{x})<h_{i \min }^{\prime}+\varepsilon_{v i} \\
0 & h_{i}^{\prime}(\mathbf{x})<h_{i \min }^{\prime} \operatorname{orh}_{i}^{\prime}(\mathbf{x})>h_{i \max }^{\prime}
\end{array}\right\}
$$

where $\varepsilon_{v i}$ is allowable maximum offset of $h_{i}^{\prime}(\mathbf{x})$.

On the basis of fuzzy set theory [1], the problem (1) is converted to maximum satisfaction degree problem [2], namely

$$
\begin{array}{ll}
\max & \\
\text { s.t. } & \mathbf{g}_{1}(\mathbf{x})=\mathbf{0} \\
\mathbf{g}_{2}(\mathbf{x})=\mathbf{0} \\
& f(\mathbf{x})+\varepsilon_{p} \cdot S \leq f_{m} \\
\mathbf{h}^{\prime}(\mathbf{x})-\boldsymbol{\varepsilon}_{v} \cdot S \geq \mathbf{h}_{\min }^{\prime} \\
\mathbf{h}^{\prime}(\mathbf{x})+\boldsymbol{\varepsilon}_{v} \cdot S \leq \mathbf{h}_{\max }^{\prime} \\
\mathbf{h}_{\min } \leq \mathbf{h}(\mathbf{x})<\mathbf{h}_{\max } \\
0 \leq S \leq 1
\end{array}
$$

where $S$ is satisfaction degree.

\section{Fuzzy Reactive Power Optimization Based on Interior Point Filter Algorithm}

The interior-point filter algorithm [9, 10] is based on the primal-dual interior-point algorithm and maintains an updating "filter" at each iteration in order to avoid oscillation when contradiction exists between the decrease of objective function and the satisfaction of constraints and ensure the global convergence. The algorithm also improves calculation efficiency due to the "filter" filters unnecessary iteration points.

To solve (4) using IPFA, inequality constraints are transformed into equality constraints by adding to slack variables and new objective function is built by introducing barrier function. Meanwhile, the problem (4) is converted to nonlinear minimizing problem, that is 


$$
\begin{aligned}
& \min \varphi_{\mu}\left(\mathbf{x}, \mathbf{x}^{\prime}, S\right)=-S-\mu\left(\sum_{i=1}^{\mathrm{N}_{\mathrm{H}}} \ln \mathbf{s}_{i l}+\sum_{i=1}^{\mathrm{N}_{\mathrm{H}}} \ln \mathbf{s}_{i u}\right. \\
& +\sum_{i=1}^{\mathrm{N}_{\mathrm{S}}} \ln \mathbf{s}_{i l}^{\prime}+\sum_{i=1}^{\mathrm{N}_{\mathrm{S}}} \ln \mathbf{s}_{i u}^{\prime} \\
& +\ln s_{s l}+\ln s_{s u}+\ln s_{f} \text { ) } \\
& \text { s.t. } \mathbf{g}_{1}(\mathbf{x})=\mathbf{0} \\
& \mathbf{g}_{2}(\mathbf{x})=\mathbf{0} \\
& f(\mathbf{x})+\varepsilon_{p} \cdot S-f_{m}+s_{f}=0 \quad s_{f} \geq 0 \\
& \mathbf{h}^{\prime}(\mathbf{x})-\boldsymbol{\varepsilon}_{v} \cdot S-\mathbf{h}_{\min }^{\prime}-\mathbf{s}_{l}^{\prime}=\mathbf{0} \quad \mathbf{s}_{l}^{\prime} \geq \mathbf{0} \\
& \mathbf{h}^{\prime}(\mathbf{x})+\boldsymbol{\varepsilon}_{v} \cdot S-\mathbf{h}_{\text {max }}^{\prime}+\mathbf{s}_{u}^{\prime}=\mathbf{0} \quad \mathbf{s}_{u}^{\prime} \geq \mathbf{0} \\
& \mathbf{h}(\mathbf{x})-\mathbf{h}_{\min }-\mathbf{s}_{l}=\mathbf{0} \quad \mathbf{s}_{l} \geq \mathbf{0} \\
& \mathbf{h}(\mathbf{x})-\mathbf{h}_{\text {max }}+\mathbf{s}_{u}=\mathbf{0} \quad \mathbf{s}_{u} \geq \mathbf{0} \\
& S-s_{s l}=0 \quad s_{s l} \geq 0 \\
& S-1+s_{s u}=0 \quad s_{s u} \geq 0
\end{aligned}
$$

where $\mathbf{x}, \mathbf{s}_{l}^{\prime}, \mathbf{s}_{u}^{\prime}, \mathbf{s}_{l}, \mathbf{s}_{u}, s_{s l}, s_{s u}$ and $s_{f}$ are the vectors composed of original variables,

$$
\mathbf{x}^{\prime}=\left[\mathbf{s}_{l}{ }^{\mathrm{T}}, \mathbf{s}_{u}{ }^{\mathrm{T}}, \mathbf{s}_{l}{ }^{\mathrm{T}}, \mathbf{s}_{u}{ }^{\mathrm{T}}, s_{s l}, s_{s u}, s_{f}\right]^{\mathrm{T}} .
$$

In IPFA, the decrease of objective function $\varphi_{\mu}\left(\mathbf{x}, \mathbf{x}^{\prime}, S\right)$ is equivalent to the satisfaction of constraints which is denoted as:

$$
\theta\left(\mathbf{x}, \mathbf{x}^{\prime}, S\right)=\left\|\begin{array}{c}
\mathbf{g}_{1}(\mathbf{x}) \\
\mathbf{g}_{2}(\mathbf{x}) \\
f(\mathbf{x})+\varepsilon_{p} \cdot S-f_{m}+s_{f} \\
\mathbf{h}^{\prime}(\mathbf{x})-\boldsymbol{\varepsilon}_{v} \cdot S-\mathbf{h}_{\min }^{\prime}-\mathbf{s}_{l}^{\prime} \\
\mathbf{h}^{\prime}(\mathbf{x})+\boldsymbol{\varepsilon}_{v} \cdot S-\mathbf{h}_{\text {max }}^{\prime}+\mathbf{s}_{u}^{\prime} \\
\mathbf{h}(\mathbf{x})-\mathbf{h}_{\min }-\mathbf{s}_{l} \\
\mathbf{h}(\mathbf{x})-\mathbf{h}_{\text {max }}+\mathbf{s}_{u} \\
S-s_{s l} \\
S-1+s_{s u}
\end{array}\right\|_{\infty}
$$

A set called "filter" is maintained at each iteration ${ }^{k}$, which is defined as:

$$
\begin{aligned}
& F_{k} \subseteq\left\{\left(\varphi_{\mu}\left(\mathbf{x}_{k}, \mathbf{x}_{k}^{\prime}, S_{k}\right), \theta\left(\mathbf{x}_{k}, \mathbf{x}_{k}^{\prime}, S_{k}\right)\right) \in R^{2}\right\} \\
& \left(\varphi_{\mu}\left(\mathbf{x}_{k+1}, \mathbf{x}_{k+1}^{\prime}, S_{k+1}\right), \theta\left(\mathbf{x}_{k+1}, \mathbf{x}_{k+1}^{\prime}, S_{k+1}\right)\right) \notin F_{k}
\end{aligned}
$$

is called if the condition

$$
\begin{aligned}
& \varphi_{\mu}\left(\mathbf{x}_{k+1}, \mathbf{x}_{k+1}^{\prime}, S_{k+1}\right)<\varphi_{\mu}\left(\mathbf{x}_{k}, \mathbf{x}_{k}^{\prime}, S_{k}\right) \\
& \operatorname{or} \theta\left(\mathbf{x}_{k+1}, \mathbf{x}_{k+1}^{\prime}, S_{k+1}\right)<\theta\left(\mathbf{x}_{k}, \mathbf{x}_{k}^{\prime}, S_{k}\right)
\end{aligned}
$$

is satisfied.

The iteration point $\left(\mathbf{x}_{k+1}, \mathbf{x}_{k+1}^{\prime}, S_{k+1}\right)$ is accepted only if objective function $\varphi_{\mu}(\mathbf{x}, \mathbf{x}, S)$ and infinite norm of the constraints set $\theta(\mathbf{x}, \mathbf{x}, S)$ meet (8).

At iteration $k+1$, the "filter" is updated according to
(9).

$$
F_{k+1}=F_{k} \cup\left\{\begin{array}{l}
(\varphi, \theta) \in R^{2}: \varphi \geq \varphi\left(\mathbf{x}_{k}, \mathbf{x}_{k}^{\prime}, S_{k}\right) \\
\text { and } \theta \geq \theta\left(\mathbf{x}_{k}, \mathbf{x}_{k}^{\prime}, S_{k}\right)
\end{array}\right\}
$$

In order to solve (5), the Lagrangian function is represented as:

$$
\begin{aligned}
L= & -S-\mu\left(\sum_{i=1}^{\mathrm{N}_{\mathrm{H}}} \ln \mathbf{s}_{i l}+\sum_{i=1}^{\mathrm{N}_{\mathrm{H}}} \ln \mathbf{s}_{i u}\right. \\
& +\sum_{i=1}^{\mathrm{N}_{\mathrm{S}}} \ln \mathbf{s}_{i l}^{\prime}+\sum_{i=1}^{\mathrm{N}_{\mathrm{S}}} \ln \mathbf{s}_{i u}^{\prime} \\
& \left.+\ln s_{s l}+\ln s_{s u}+\ln s_{f}\right) \\
& -\mathbf{y}_{1}^{\mathrm{T}} \mathbf{g}_{1}(\mathbf{x})-\mathbf{y}_{2}{ }^{\mathrm{T}} \mathbf{g}_{2}(\mathbf{x}) \\
& -\mathbf{y}_{l}{ }_{l}^{\mathrm{T}}\left(\mathbf{h}^{\prime}(\mathbf{x})-\boldsymbol{\varepsilon}_{v} \cdot S-\mathbf{h}_{\text {min }}^{\prime}-\mathbf{s}_{l}^{\prime}\right) \\
& -\mathbf{y}_{u}{ }^{\mathrm{T}}\left(\mathbf{h}(\mathbf{x})+\boldsymbol{\varepsilon}_{v} \cdot S-\mathbf{h}_{\max }^{\prime}+\mathbf{s}_{u}^{\prime}\right) \\
& -\mathbf{y}_{l}{ }^{\mathrm{T}}\left(\mathbf{h}(\mathbf{x})-\mathbf{h}_{\min }-\mathbf{s}_{l}\right) \\
& -\mathbf{y}_{u}{ }^{\mathrm{T}}\left(\mathbf{h}(\mathbf{x})-\mathbf{h}_{\max }+\mathbf{s}_{u}\right) \\
& -\mathrm{y}_{s l}\left(S-s_{s l}\right)-\mathrm{y}_{s u}\left(S-1+s_{s u}\right) \\
& -\mathrm{y}_{f}\left(f(\mathbf{x})+\varepsilon_{p} \cdot S-f_{m}+s_{f}\right)
\end{aligned}
$$

where $\mathbf{y}_{1}, \mathbf{y}_{2}, \mathbf{y}_{l}^{\prime}, \mathbf{y}_{u}^{\prime}, \mathbf{y}_{l}, \mathbf{y}_{u}, \mathrm{y}_{s l}, \mathrm{y}_{s u}$ and $\mathrm{y}_{f}$ are the vectors composed of dual variables.

According to Karush-Kuhn-Tucker condition, it is the necessary condition that all the partial derivatives of the Lagrangian function are equal to zero if the minimum of the problem (5) is existed. Derivation process and solution procedure for using the IPFA to solve the nonlinear programming model can be referred to [11].

\section{Case Study}

Test case of a practical 244-bus power system is employed to validate the solution of fuzzy model for reactive power optimization based on interior-point filter algorithm. The system contains 76 generation units, 12 on-load tap changers and 56 shunt capacitor/reactors. The current controllable devices include 36 generation units, 12 on-load tap changers and 52 shunt capacitor/reactors. The computer configuration used for case study is Intel Core i3 $2.53 \mathrm{GHz}$ and 2 GB memory.

In this case, the reference power is 100 MVA; the lower and upper limits of bus voltages are set at 0.9 and 1.1 (p.u.); there are 12 buses violated voltage limits in the initial state; the initial network loss is 1.2516 (p.u.). $\varepsilon_{p}$ and the elements of vector ${ }^{\varepsilon_{v}}$ are set to 0.233 and 0.0217 respectively. After optimization calculation for fuzzy model, the satisfaction degree is 0.9689 , all bus voltages are within limits and the network loss is reduced to 1.0258. Comparisons of optimal results and the initial values are in Table 1. Moreover, comparisons of solving results of fuzzy model and traditiona1 model for reactive 
Table 1. Comparisons of optimal results and the initial values.

\begin{tabular}{lcc}
\hline & $\begin{array}{c}\text { Initial } \\
\text { values }\end{array}$ & $\begin{array}{c}\text { Optimal } \\
\text { results }\end{array}$ \\
\hline network loss(p.u.) & 1.2516 & 1.0258 \\
number of buses violated voltage limits & 12 & 0 \\
maximum voltage of PQ bus(p.u.) & 1.0571 & 1.0789 \\
minimum voltage of PQ bus (p.u.) & 0.8927 & 0.9211 \\
\hline
\end{tabular}

Table 2. Comparisons of solving results of fuzzy model and traditional model.

\begin{tabular}{lcc}
\hline & $\begin{array}{c}\text { fuzzy } \\
\text { model }\end{array}$ & $\begin{array}{c}\text { traditiona1 } \\
\text { model }\end{array}$ \\
\hline network loss(p.u.) & 1.0258 & 1.0183 \\
number of buses violated voltage limits & 0 & 0 \\
maximum voltage of PQ bus(p.u.) & 1.0789 & 1.1 \\
minimum voltage of PQ bus (p.u.) & 0.9211 & 0.9225 \\
computing time(s) & 0.95 & 0.59 \\
\hline
\end{tabular}

power optimization are shown in Table 2.

From the Table 1 and Table 2, it can be seen that the algorithm can effectively deal with the large number of inequality constraints of the fuzzy model of reactive power optimization and has high compute efficiency. After optimization calculation, there is no bus violated voltage limit through the adjustment of the reactive power controllers. The maximum voltage of PQ bus is its upper limit when traditiona1 model is used, however, the voltages of PQ buses maintain specified margins when fuzzy model is used, which improves the safety level of the system.

Meanwhile, the network loss is decreased to 1.0183 from 1.2516 and the decreasing range is $18.63 \%$ when traditiona1 model is used, whereas the network loss is decreased to 1.0258 from 1.2516 and the decreasing range is $18.04 \%$ when fuzzy model is used. So the decreasing range of network loss obtained by solving the fuzzy model is less than that obtained by solving the traditiona1 model, but the level of voltage security is improved when fuzzy model is used.

The example of the practical power system indicates that the algorithm can effectively solve the fuzzy model of reactive power optimization and satisfy the requirement of online calculation. After optimization calculation, the violated voltages are corrected; the voltage soft constraint buses are kept specified margins; at the same time the network loss is reduced.

\section{Conclusions}

This paper uses the interior point filter algorithm to solve the formulation of fuzzy model for the power system reactive power optimization considering the soft constraint characteristics of voltage constraints. Optimization results show that the algorithm can effectively deal with the large number of equality and inequality constraints of the fuzzy model for the practical power system and satisfy the requirement of online calculation which realizes to decrease the network loss and maintain security margins of voltage.

\section{REFERENCES}

[1] H. Yuan, G. G. Xu and J. Y. Zhou, “A Reactive Power/voltage Optimization Based on Fuzzy Linear Programming,” Power System Technology, Vol. 27 , No. 12, 2003, pp. 42-45.

[2] F. R. Tu and X. R. Wang, "Fuzzy Modeling for Power System Reactive Power Optimization,” Power System Protection and Control, Vol. 38, No. 13, 2010, pp. 46-49.

[3] Y. N. Li, L. Z. Zhang and Y. H. Yang, "Reactive Power Optimization Under Voltage Constraints Margin,” Proceedings of the CSEE, Vol. 21, No. 9, 2001, pp. 1-4.

[4] K. Tomsovic, "A Fuzzy Linear Programming Approach to the Reactive Power/Voltage Control Problem," IEEE Trans on Power Systems, 1992, Vol. 7, No. 1, pp. 287-293. doi:10.1109/59.141716

[5] M. B. Liu, Y. Cheng and S. H. Lin, "Comparative Studies of Interior-point Linear and Nonlinear Programming Algorithms for Reactive Power Optimization," Automation of Electric Power Systems, Vol. 26, No. 1, 2002, pp. 22-26.

[6] M. B. Liu, S. K. Tso, Y. Cheng, “An Extended Nonlinear Primal-dual Interior-point Algorithm for Reactive-power Optimization of Large-scale Power Systems with Discrete Control Variables,” IEEE Trans on Power Systems, Vol. 17, No. 4, 2002, pp. 982-991. doi:10.1109/TPWRS.2002.804922

[7] J. D. Xu, X. Q. Ding, Z. C. Qin Zhencheng, et a1., “A Nonlinear Predictor-Corrector Interior Point Method for Reactive Power Optimization in Power System," Power System Technology, Vol. 29, No. 9, 2005, pp. 36-40.

[8] K. Pan, X. S. Han and X. X. Meng, Solution Principles Study of Nonlinear Correction Equations in Primal-dual Interior Point Method for Reactive Power Optimization," Power System Technology, Vol. 30, No. 19, 2006, pp. 59-65.

[9] U. Michael, S. Ulbrich, L. N. Vicente, A Globally Convergent Primal-dual Interior-point Filter Method for Nonlinear Programming," Mathematical Programming, Vol. 100, No. 2, 2004, pp. 379-410. doi:1007/s10107-003-0477-4

[10] W. Andreas and L. T. Bieglery, "Line Search Filter Methods for Nonlinear Programming: Motivation and Global Convergence,” Yorktown Heights, USA: IBM T. J. Watson Research Center, 2001. 
[11] S. Yang, J. Y. Zhou, Q. Li, et a1., "An Interior-point Reactive Power Optimization Based on Filter Set," Power System Protection and Control, 2011, Vol. 39, No. 18, pp. 14-19.
[12] Y. Y. Sun, G. Y. He and S. W. Mei, "A New Optimal Power Flow Algorithm Based on Filter Interior Point Method,” Advanced Technology of Electrical Engineering and Energy, Vol. 26, No. 2, 2007, pp. 29-33. 